\title{
Zur Entwicklung ärztlicher Führung
}

\author{
Dr. Christof Schmitz ${ }^{a}$, Prof. Dr. Matthias Egger ${ }^{b}$, PD Dr. Peter Berchtold ${ }^{a}$ b
}

${ }^{a}$ college M, Bern; ${ }^{b}$ Institut für Sozial- und Präventivmedizin, Universität Bern

\begin{abstract}
Auf den Punkt gebracht entwickeln sich Spitäler und mit ihnen die organisierte Krankenbehandlung dramatisch: Diagnostische und therapeutische Möglichkeiten und mit ihnen Spezialisierung und Technologisierung nehmen rasant zu. Ökonomisierung, sich wandelnde Erwartungen der Patienten, der Mitarbeitenden und anderer Stakeholder wie Politik und Medien steigern weiter die Komplexität. Ärztliche Führung lässt dies nicht unberührt, sie hat den Übergang hin zu einer Leadership zu meistern, die den neuen, komplexen Verhältnissen gerecht(er) wird.
\end{abstract}

\begin{abstract}
Spitäler Anfang der 1980er Jahre waren noch Orte grosser Einfachheit im Vergleich zu dem, was wir heute vorfinden (Abb. 1). Spezialisierung fand sich wenig ausgeprägt, vielfach war mit einer medizinischen, einer chirurgischen und vielleicht noch einer geburtshilflichen Klinik Genüge getan. Im Spital hatten vor allem die Ärzte das Sagen, sie verfügten über das Gros des Entscheidungskapitals. Der Verwaltungsdirektor tat das, was sein Name ausdrückte, nämlich verwalten, und das, was die Ärzte ihm sagten. Die Patienten und Patientinnen standen den Ärzten noch unkritisch gegenüber, und die Fachgesellschaften - als «heimliche Vorgesetzte» - gaben die Richtungen vor, in die es zu gehen galt.
\end{abstract}

Mit den 90er Jahren wurde die Spezialisierung der Medizin prägnanter spürbar, etwa mit der Zersplitterung der Inneren Medizin, indem sich die Kardiologie und

CAS Leadership in Organisationen des Gesundheitssystems

Führungskompetenz im medizinischen Kerngeschäft ist erfolgskritisch geworden. Das ist heute evidenzbasiert. Der Studiengang «CAS Leadership in Organisationen des Gesundheitssystems» vermittelt speziell eine an Organisationen des Gesundheitswesens und ihren Entwicklungen orientierte Leadership. Das Programm verbindet evidenzbasiertes Wissen mit praktischen Anwendungen in einer anregenden Lernkultur.

Der Studiengang wird vom Institut für Sozial- und Präventivmedizin der Universität Bern und vom college M durchgeführt. Er richtet sich an Ärzte und Ärztinnen mit leitenden Funktionen sowie Führungskräfte anderer Berufsgruppen in Spitälern und weiteren Einrichtungen.

Der Studiengang gliedert sich in 6 Module von insgesamt 18Tagen und eine Projektarbeit. Der Studiengang wird mit einem «Certificate of Advanced Studies (CAS) in Leadership in Health Care Organisations» der Universität Bern (15 ECTS-Punkte) abgeschlossen. Detaillierte Informationen unter www.cas-leadership.ch oderTel. 0316313511. andere Spezialisten herauslösten und «organorientierten» Verbünden den Vorzug gaben. Höhere Aufmerksamkeit als bis anhin beanspruchten auch ökonomische Themen, Ressourcen zeigten sich erstmals als nicht beliebig verfügbar. Und an der Spitze der Spitäler tauchten die ersten Betriebswirte auf. Zwischen Medizin und Management begann der Streit um das Entscheidungskapital.

\section{Steigende Komplexität}

Diese Dynamik akzentuierte sich in den Ooer Jahren. Die Ökonomie und mit ihr die Betriebswirte gewannen weiter an Bedeutung, Managementmethoden fassten zunehmend Fuss, Qualitätsmanagement, Balanced Scorecards und dergleichen hielten Einzug, Bereichsbildungen und Departementalisierungen pflügten Organigramme um, und Controller beanspruchten Führungsgeltung. Im Kampf darum, wer das Sagen hat, war die nächste Runde eingeläutet. An der Spitze der Spitäler tauchten die ersten CEOs auf - typischerweise keine Ärzte.

Mit den 10er Jahren entfaltete sich die Spezialisierung weiter, ablesbar an der mittlerweile auf weit über 100 angestiegenen Anzahl an Fach- und Fähigkeitstiteln. Die Anzahl Schnittstellen pro Patient stieg rasant, wie Atul Gawande, Chirurg in Harvard, treffend aufzeigte: Waren in den 70ern noch 2,5 Vollzeitäquivalente für die Behandlung eines durchschnittlichen Patienten nötig, brauchte es nun gegen 50,1. Das erhöhte den Aufwand an Abstimmung und Zusammenarbeit gewaltig und weckte das Interesse an integrierenden Modellen, wie z.B. Zentren-Bildung an Spitälern. Mit der Einführung der DRGs 2012 wurde ein nächster Schritt der Ökonomisierung unternommen, die Hand in Hand mit einer weiteren «Verbetriebswirtschaftlichung» des 


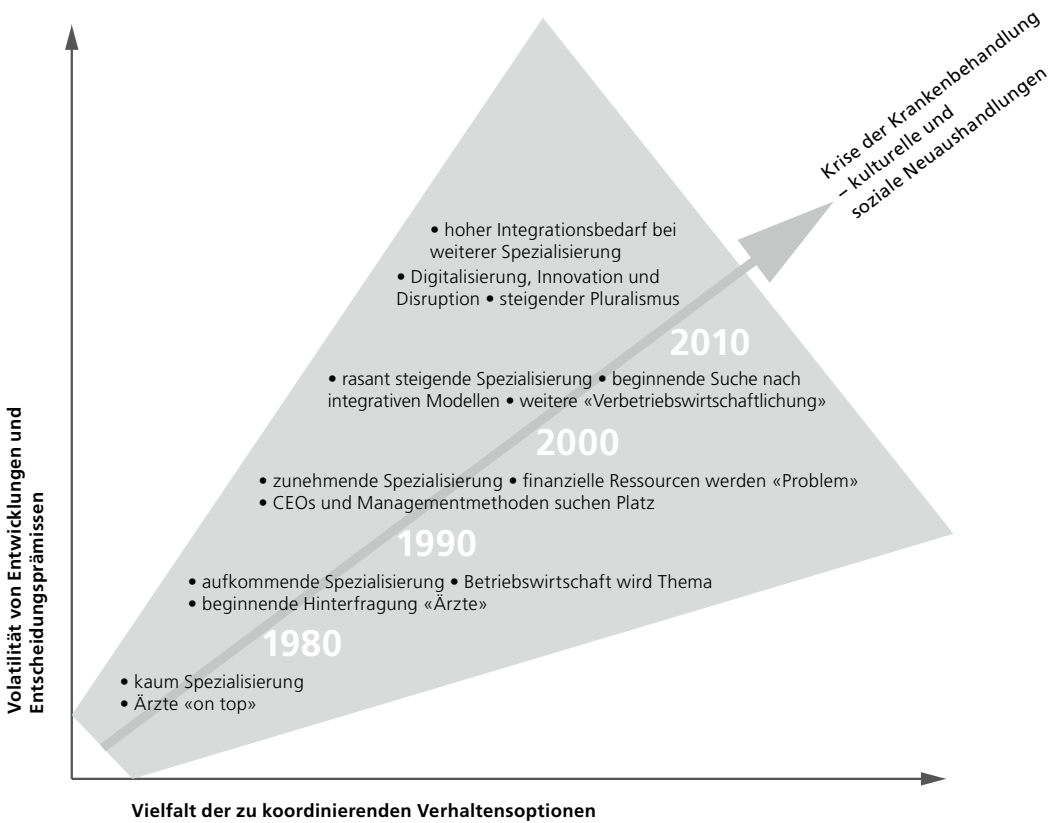

Abbildung 1: Landkarte der Spitalentwicklung (Achsen adaptiert nach Heitger/Serfass 2015) [2]. etc. und die zunehmende gesellschaftliche Brisanz dieser Entwicklungen (Datenschutz etc.), die ihrerseits Erwartungen und Ansprüche der Patientinnen und Patienten, der Öffentlichkeit und der Politik unterschiedlicher und unberechenbarer werden lassen. In einem Satz: Neben der Vielfalt werden sich auch die Volatilitäten der Entwicklungen nochmals erheblich vergrössern (Ordinate in Abb. 1). Die Rollenbilder der Medizin-, Pflege- und Gesundheitsberufe wie auch ganzer Organisationen werden damit noch mehr in Fluss geraten. Von der Eindimensionalität - it's about medicine, stupid; um einen alten Spruch zu bemühen geht die Reise hin zur Pluralität der zu berücksichtigenden Kontexte.

\section{Jenseits des Heroismus}

Im Angelpunkt dieser Dynamiken finden sich Führung und Führungspersonen wieder. Oftmals zeigt sich die nötige Diskussion dieser Dynamiken überlagert vom allgegenwärtigen «Verbetriebswirtschaftlichungs-Diskurs», der Spitäler seit Jahren fest im Griff hält: Etwas verkürzt sollen Ärzte und Ärztinnen management-like denken und handeln, das meint im Kern zahlenorientiert agieren und strukturiert und effizient Routinen abarbeiten, etwa im Stile des klassischen "plan-do-check-act»-Zyklus. So wie dieses Konzept sind viele Managementmethoden für einfache bzw. potentiell standardisierbare Kontexte gedacht und bieten nur unzureichende Hilfestellung in komplexen Verhältnissen, so auch denen der künftigen Krankenbehandlung. Dann tendieren betriebswirtschaftliche Diskurse dazu, die brisante Frage, wie sich die ärztliche Führung zu verändern hat, um die mehrdimensionalen medizinischen und gesundheitssystemischen Themen der Zukunft erfolgreich bewältigen zu können, eher zu vernebeln als anzureichern. der Wandlungsprozess, in dem sich das soziale und kulturelle Verständnis von Gesundheit und Krankenbehandlung befindet. Nimmt man die Spannweite von Wellness, Anti-Aging u.ä. auf der einen und neue (Labor-)Technologien wie CrispR auf der anderen Seite hinzu, wird unmittelbar ersichtlich, mit welcher Vielfalt und welcher Unübersichtlichkeit in der Krankenbehandlung man heute zu tun hat (Abszisse in Abb. 1).

\section{Ärzte haben heute nicht mehr "nur» Medizin zu betreiben, sondern sollen zugleich betriebs- wirtschaftliche Erfolge leisten.}

Ein Blick in die Zukunft informiert rasch darüber, dass sich diese Komplexität weiter steigern wird. Dafür sorgen fortschreitende Technologisierung, Digitalisierung

\section{Neben der Vielfalt werden sich auch die Volatilitäten der Entwicklungen nochmals erheblich vergrössern.}

Führen heisst Einfluss nehmen, und Einfluss kann in unterschiedlichster, positiver wie auch negativer, Hinsicht ausgeübt werden: War klassische Medizin lange und zunächst gut passend von einer Kultur des heroic leadership gekennzeichnet - der Chefarzt oder der Ordinarius, der einsam aber status- und machtbewusst und natürlich unermüdlich für seine Klinik, seine Patienten, seine Betten kämpfte - , verlangt die neue Welt ein SichEinbringen in vernetzte, oft fluide, netzwerkartige Strukturen und Prozesse, die von Kollaboration und wechselseitigen Abhängigkeiten geprägt sind und die einsames 


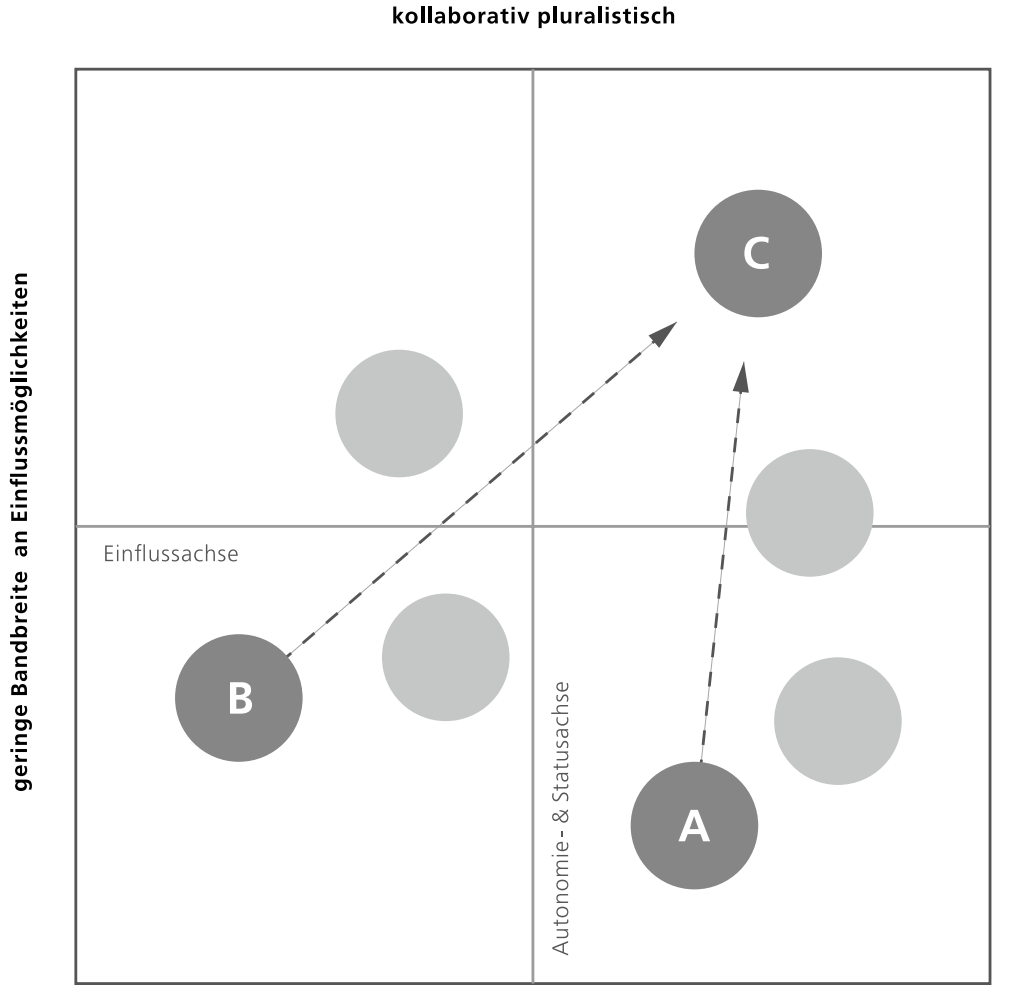

individualistisch eindimensional

Abbildung 2: Landkarte der ärztlichen Führung (eigene Darstellung).

Heldentum schlecht vertragen. Dem alten Hauen und Stechen im Kampf um das eigene Territorium - «Diese Patientin gehört mir!» - steht das Zusammenspiel mit anderen diametral gegenüber. Natürlich ist das eine Überzeichnung. Sie soll die diesbezüglichen Positionen entlang einer Autonomie- und Statusachse von individualistisch vs. kollaborativ klären (Abb. 2).

\section{Ärztliche Führung heisst Einfluss nehmen, wie Krankenbehandlung organisiert und gestaltet wird, wie Patienten und Patientinnen behan- delt und Mitarbeitende geführt werden.}

Ärztliche Führung heisst Einfluss nehmen, wie Krankenbehandlung organisiert und gestaltet wird, wie Patienten und Patientinnen behandelt und Mitarbeitende geführt werden. Dafür kennen wir unterschiedliche Formen: fachlichen Input, Inspiration, Gestaltung von Kommunikation und ihren Gefässen und entsprechenden Organisationsstrukturen, das Entwickeln und Fördern von Mitarbeitenden, das Pflegen vertrauensvoller Zusammenarbeit, aber auch das Setzen der Agenda in den entscheidenden Meetings, das Flechten von Abhängigkeitsbeziehungen und anderen Formen des Powerplays. Einflussnahme erschöpft sich auch in Zukunft nicht in den Mitteln so- genannter guter Führung [3]. Das eine ist mit Blick auf Führungserfolg empfehlenswerter als das andere, eine gewisse Bandbreite existiert immer. Falsch wäre, den Blick auf die Realität unangemessen zu verengen. Auch die neuen, kollaborativen Führungswelten sind nicht frei von Macht- und Statusfragen, und Machtressourcen wie Patientenströme, Erträge, Reputation etc. bieten weiter ihre verführerischen Optionen. Ein produktiver und gestalterischer Umgang mit Macht ist gefragt. So kann eine Prise Macht helfen, Verständigungsbereitschaft zu erhöhen, zu viel davon kann aber auch Vertrauen beschädigen. Wichtig wäre eine Auseinandersetzung darüber nicht nur auf individueller, sondern auch auf Ebene der Organisationen.

\section{Zwischen Skylla und Charybdis}

Mediziner und Medizinerinnen unterscheiden sich unserer Erfahrung nach darin, welche Vielfalt an Einflussoptionen sie wahrzunehmen wissen. Der Anschaulichkeit halber stellen wir simplifizierend gegenüber: Da sind zum einen diejenigen, die kreativ immer wieder neue Möglichkeiten der Einflussnahme finden, während andere nur wenig Handlungsräume zu nutzen wissen. Wir können diese Polarität ebenfalls entlang einer (Einfluss-)Achse anordnen und orthogonal zur vorherigen Autonomie- und Statusachse zeichnen.

Das dabei entstehende Vier-Felder-Schema erlaubt eine grobe Verortung idealtypischer, medizinischer Führungspositionen (Abb. 2). Die Position A bezeichnet jene, die eine breite Klaviatur an Einflussnahmen spielen können, aber in klassisch-ärztlicher Manier den Mollakkorden von Macht und Status verhaftet sind. Position B bezeichnet jene, die ähnlich geprägt sind, aber weniger Handlungsräume zu nutzen wissen. Beide Positionen drohen in der neuen ärztlichen Führungswelt nicht mehr zu genügen, beide werden angesichts der beschriebenen Komplexitätssteigerung in Richtung von Position C gedrängt. Sind die Positionen A und B noch stark im Kampf um Autonomie und Status gefangen, bezeichnet $\mathrm{C}$ eine Position, die daran orientiert ist, gemeinsam mit anderen Lösungen in mehrdimensionalen Verhältnissen zu finden und da-

\section{Die Realität in den Spitälern heute ist von der Ungleichzeitigkeit der Führungsverständnisse gekennzeichnet.}

durch den eigenen Einfluss zu steigern. In diesem Feld ist dann weniger die Frage, ob dieser Patient «mir gehört» (Macht/Autonomie) oder ob das «meine Betten sind» (Status), denn wie sich Spezialitäten zugunsten 


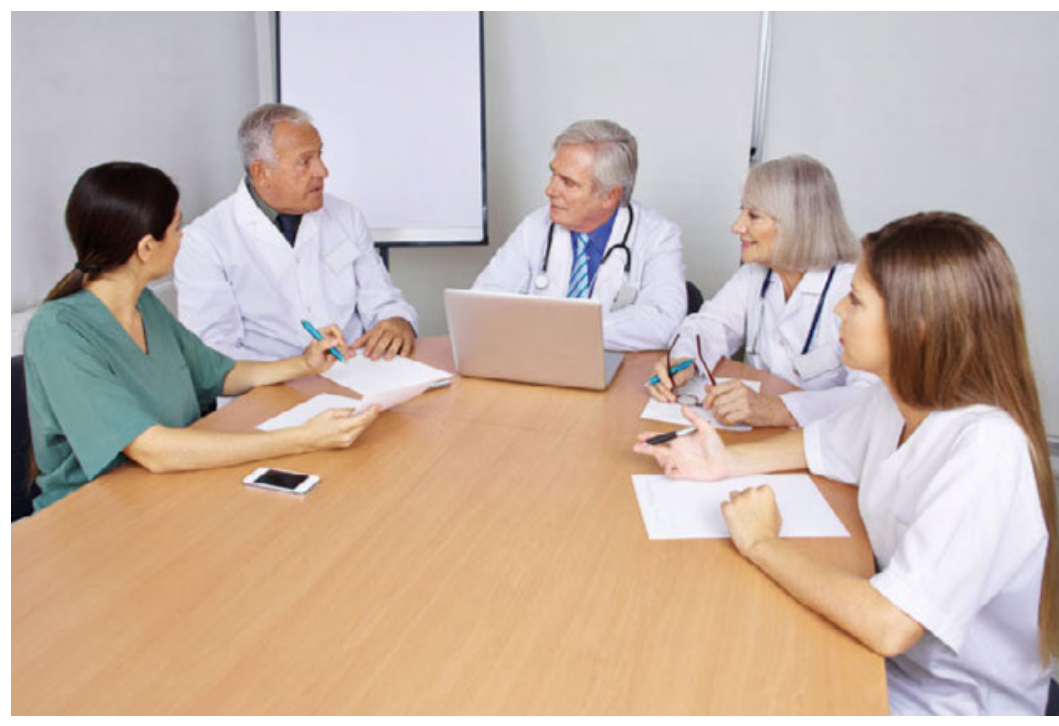

Angesichts der sich wandelnden Erwartungen von Patienten und Mitarbeitern, neuer diagnostischer und therapeutischer Möglichkeiten sowie der Ökonomisierung der Medizin müssen Ärzte lernen, anders zu führen (Symbolbild).

von Patientinnen und einer besseren Versorgung kombinieren können.

Die Realität in den Spitälern heute ist von der Ungleichzeitigkeit der Führungsverständnisse gekennzeichnet. Da finden sich Relikte des alten Kampfes um Autonomie und klassische Beispiele der «Kunst der Territorialverteidigung» («gekonnt geturft») neben luziden Momenten neuer Vernetzungsformen, beispielsweise wenn beim zusatzversicherten Patienten noch der Zweihänder ausgepackt wird, während man im
Tumorboard auf Augenhöhe und im besten Sinne kollegial miteinander arbeitet. Die Entwicklung hin zu einem kollaborativen Führungsverständnis wird, wie erwähnt, vom «Verbetriebswirtschaftlichungs-Diskurs» gelegentlich eher behindert denn befördert. Das Ziel ist nicht, dass Ärzte und Ärztinnen sich als Manager aufzurüsten hätten. Auch Manager haben aufzurüsten. Medizinische, versorgungs- und patientenbezogene Fragestellungen der Zukunft erschöpfen sich nicht in Themen der Ökonomie, also der Bewirtschaftung knapper Ressourcen, so wichtig diese auch sind, sondern brauchen den ganzen (medizinischen, ökonomischen, ethischen, emotionalen ...) Verstand, um der neuen Welt der Spezialisierung wie der Integration, der Individualisierung sowie der kritischen Beobachtung der Gesellschaft erfolgreiche Lösungen zugunsten eines nachhaltigen Gesundheitssystems abringen zu können. Dieser medizinische Verstand ist nicht mehr im Kopf des lonesome hero (Position A) zu finden, sondern ist nur mehr als ein Viel-Hirn-Phänomen (Position C) vorstellbar.

\section{Literatur}

1 Gawande A (2011). Cowboys and Pit Crews. The New Yorker, May 26, 2011.

2 Heitger B / Serfass A (2015). Unternehmensentwicklung. Wissen, Wege, Werkzeuge für morgen. Schäffer-Poeschel.

3 Schmitz C, Egger M, Berchtold P (2016). Leadership-Bullshit. Schweiz Ärztezeitung. 97(36):1222-4.

Bildnachweise

Abbildungen 1 und 2: college $M$

Symbolbild: @ Stylephotographs | Dreamstime.com 